\title{
Efficacité des traitements chimique et biologique sur les Culicidae : effet létal du téméphos et taux de consommation par Gambusia affinis
}

\author{
S. Bouallam ${ }^{1}$ \\ A. Maarouf ${ }^{2}$ \\ A. Bouzidi ${ }^{3}$ \\ A. Badri ${ }^{1}$
}

Mots clés : lutte chimique, téméphos (organophosphoré), lutte biologique, Gambusia affinis, paludisme, Culicidae, Anopheles hispaniola, Culex pipiens.

Dans ce travail, la lutte chimique anti-larvaire a été réalisée au laboratoire chez deux espèces culicidiennes Anopheles hispaniola, vecteur potentiel du paludisme (Guy 1963), et Culex pipiens. Dans ces essais, nous avons utilisé le téméphos, larvicide organophosphoré, sous forme liquide $500 \mathrm{EC}$ (Concentration Emultionnable). Dans ces conditions, un litre de produit commercial contient $500 \mathrm{~g}$ de produit actif $\left(500 \mathrm{~g} \cdot \mathrm{l}^{-1}\right)$.

Cette étude a montré que la $\mathrm{CL}_{50}(30 \mathrm{mn})$ pour le stade L2 est de $21 \mu \mathrm{g} . \mathrm{l}^{-1} \mathrm{chez}$ A.hispaniola et de $10 \mu \mathrm{g} . \mathrm{l}^{-1} \mathrm{chez} C$. pipiens ; $\mathrm{La} \mathrm{CL}_{50}(60 \mathrm{mn})$ au stade L4 est respectivement de $23 \mu \mathrm{g} . \mathrm{l}^{-1}$ et $12 \mu \mathrm{g} . \mathrm{.}^{-1}$.

Le taux de consommation des larves d'A.hispaniola (stade L4) par Gambusia affinis varie en fonction des milieux étudiés mais reste toujours plus élevé chez les femelles que chez les mâles.

Efficiency of chemical and biological treatments on Culicidae larvae: lethal effect of temephos and consumption rate by Gambusia affinis

Keywords : chemical control, temephos (phosphoorganic), biological control, Gambusia affinis, malaria, Culicidae, Anopheles hispaniola, Culex pipiens.

In this work, we carried out a laboratory study on chemical control of larvae of two species of Culicidae : Anopheles hispaniola, a potential vector of malaria (Guy 1963) and Culex pipiens using Temephos, an organo-phosphorus liquid-state EC500 (Emultion Concentation) larvicide. Under these conditions one litre of Temephos contains $500 \mathrm{~g}$ of active product (500 g.L $\mathrm{L}^{-1}$ ).

This study showed that the $\mathrm{LC}_{50}$ (30min.) at $\mathrm{L} 2$ instar was $21 \mu \mathrm{g} . \mathrm{l}^{-1}$ in $A$. hispaniola and $10 \mu \mathrm{gg} \mathrm{l}^{-1}$ in $C$. pipiens. The LC 50 (60min.) at L4 instar was $23 \mu \mathrm{g} .1^{-1}$ and $12 \mu \mathrm{g} . \mathrm{l}^{-1}$ respectively.

Consumption rate of A.hispaniola larvae (instar L4) by the mosquitofish Gambusia affinis varies according to the media but remains higher in females than in males.

\section{Introduction}

Ce travail, réalisé pour la première fois au Maroc, a pour objectif d'optimiser les moyens de lutte chimique et biologique en testant les effets respectifs du témé-

\footnotetext{
1. Laboratoire d'Hydrobiologie, Département de Biologie, Faculté des Sciences Semlalia, B.P. S.15, Marrakech, Maroc.

2. Laboratoire de Biosurveillance, Département de Biologie, Faculté des Sciences \& Techniques, Guéliz, B.P. 618, Marrakech, Maroc. 3. Laboratoire de Sciences de l'Environnement, Département de Biologie, Faculté des Sciences \& Techniques, B.P. 577, Settat, Maroc.
}

phos et de Gambusia affinis (Baird \& Girard, 1853) sur la croissance et la mortalité des larves de moustiques (Culicidae) : Anopheles hispaniola (Théobald,1903), vecteur potentiel du paludisme (Guy 1963) et Culex pipiens (Linné, 1758).

La lutte antilarvaire constitue un complément précieux à la lutte contre le vecteur du paludisme (Kline et al. 1994, Le Sueur et al. 1993, Sharp et al. 1993, Sinegre et al. 1976, Tietze et al. 1993, Vanessen et al. 1994, Vulvule et al. 1994 et Zaim et al. 1993). Dans une première partie, nous avons évalué la toxicité létale du téméphos (larvicide organophosphoré) vis-à-vis des larves de Culicidés. 
Ce travail est complété en deuxième partie par une substitution des larvicides chimiques qui comportent toujours un risque de contamination de l'environnement, par l'emploi de poissons (Gambusia affinis Baird \& Girard, 1853) particulièrement friands des larves de moustiques. La réalisation des tests avec ce poisson est considérée comme moyen de lutte biologique antipaludéenne par le Ministère marocain de la santé publique (1981) et par Teton (1976). Ainsi, plusieurs auteurs (Al-Daham et al. 1977, Hall et al. 1970, Hurlbert et al. 1972b, Hurlbert \& Mulla 1981, Krumholz 1948, Labounty \& Deacon 1972, Losos \& Hetesa 1973, Sicault 1934) ont concentré leurs efforts sur cette prédation des larves de moustiques par les poissons.

\section{Matériels et méthodes}

\subsection{Milieux d'études}

Les observations expérimentales ont été faites au laboratoire. Le matériel biologique a été récolté dans trois stations situées dans la région de Marrakech.

A.hispaniola a été récoltée dans la station Agadir Tachraft située sur l'oued N'fis. La température de l'eau est supérieure à $12^{\circ} \mathrm{C}$ en janvier et ne dépasse pas $32^{\circ} \mathrm{C}$ en juillet. Le $\mathrm{pH}$ reste faiblement alcalin, il varie entre 6,5 et 8,1 . La conductivité oscille entre 310 et 4900 $\mathrm{ms} . \mathrm{cm}^{-1}$. Les teneurs en chlorures sont comprises entre $213 \mathrm{mg} . \mathrm{l}^{-1}$ en période des crues et $603,5 \mathrm{mg} . \mathrm{l}^{-1}$ en période d'étiage. Les matières en suspension sont faibles $\left(2,01 \mathrm{mg} . \mathrm{l}^{-1}\right)$ mais peuvent atteindre des valeurs très élevées lors des crues : $1229 \mathrm{mg} . \mathrm{l}^{-1}$ (Tableau 1).

C.pipiens est récolté dans le deuxième bassin de lagunage situé dans la zone d'épandage de Marrakech (déversoir de toutes les nuisances de la ville : eaux usées, ordures ménagères). La température de l'eau du bassin varie entre $12^{\circ}, 5 \mathrm{C}$ en hiver et $31,5^{\circ} \mathrm{C}$ en été. Le $\mathrm{pH}$ est basique $(7,6$ et 8,5$)$. Le taux d'oxygène est très faible, il varie de 0 à $30 \mathrm{mg} . \mathrm{l}^{-1}$ (Tableau 1 ).

G.affinis est prélevé dans le bassin de la khettara Aourrad située dans la zone d'épandage des eaux usées de Marrakech. L'eau de cette station se caractérise par une température supérieure à $11^{\circ} \mathrm{C}$ en janvier et ne dépassant pas $32^{\circ} \mathrm{C}$ en juillet. Le $\mathrm{pH}$ varie entre 6,5 et 7,8 et la conductivité entre 1500 et $2800 \mathrm{~ms}^{\mathrm{cm}}{ }^{-1}$ (Tableau 1).

En plus de ces trois stations et, pour les expérimentations au laboratoire, l'eau a été prélevée dans un puits situé à la Faculté des Sciences Semlalia Marrakech. Ce puits se caractérise par un $\mathrm{pH}$ légèrement basique $(6,5$ à 7,3$)$ et une température de l'eau comprise entre 15 et $30^{\circ} \mathrm{C}$ (Tableau 1).

\subsection{Lutte antilarvaire chez les Culicidés}

Pendant 24 heures avant chaque expérience, les larves sont acclimatées dans l'eau du puits à la température ambiante du laboratoire ( entre 25 et $28^{\circ} \mathrm{C}$ ) pendant le mois de mai 1996 afin d'éliminer les individus fragilisés pendant le transport.

Trois tests préliminaires ont été effectués avant le test définitif. Ce dernier a été réalisé pour calculer la concentration létale pour $50 \%$ d'individus $\left(\mathrm{CL}_{50}\right)$ par la méthode des probits. Pour valider le test, la mortalité chez le témoin doit être inférieure ou égale à $10 \%$.

\subsubsection{Lutte chimique: effet létal du téméphos}

Le téméphos utilisé est sous forme liquide $500 \mathrm{EC}$ (Concentration Emulsionnable). Sous cette formula-

Tableau 1. Paramètres physico-chimiques (minimum et maximum) des eaux des différentes stations étudiées.

Table 1. Physico-chemical parameters (min. and max.) of waters from the different stations studied.

\begin{tabular}{|c|c|c|c|c|}
\hline $\begin{array}{l}\text { Paramètres } \\
\text { physico-chimiques }\end{array}$ & Agadir Tachraft & $\begin{array}{l}\text { Lagune } \\
\text { (bassin2) }\end{array}$ & $\begin{array}{l}\text { Khettara Aourrad } \\
\text { (bassin2) }\end{array}$ & Puits \\
\hline Température de l'eau $\left({ }^{\circ} \mathrm{C}\right)$ & $12,5-32$ & $12,5-31,5$ & $12,5-32$ & $15-30$ \\
\hline $\mathrm{pH}$ & $6,5-8,05$ & $7,6-8,5$ & $6,5-7,8$ & $6,5-7,5$ \\
\hline $\mathrm{O} 2\left(\mathrm{mg} \cdot \mathrm{l}^{-1}\right)$ & $4,8-18,1$ & $0-30$ & $7,6-10,3$ & $4,5-15$ \\
\hline Cond. $\left(\mu \mathrm{s} . \mathrm{cm}^{-1}\right)$ & $310-4900$ & $1100-1600$ & $1500-2800$ & $600-2000$ \\
\hline $\operatorname{MES}(\mu$ s.mg.1-1) & $2,01-1229$ & $50-200$ & $1,7-32,4$ & $1,95-25$ \\
\hline $\mathrm{Cl}-\left(\mathrm{mg} \cdot \mathrm{l}^{-1}\right)$ & $213-603,5$ & $325-600$ & $101-710$ & $210-400$ \\
\hline Dureté totale (mg.1-1) & $80-190$ & $129-416,5$ & $540-760$ & $410-520$ \\
\hline
\end{tabular}


tion, 1 litre de produit commercial contient $500 \mathrm{~g}$ de produit actif $\left(500 \mathrm{~g} \cdot \mathrm{l}^{-1}\right)$. Il est testé pour les stades L2 et L4 de Culicidae à des taux différents afin de déterminer la $\mathrm{CL}_{50}$ pendant un temps d'exposition déterminé auparavant .

Les expériences sont réalisées à raison de 5 larves par pilulier. L'essai est répété 3 fois. Les dilutions sont effectuées avec l'eau du puits tout en déterminant les paramètres physico-chimiques. Le volume final d'eau est de $50 \mathrm{ml}$ et les piluliers sont placés dans un bainmarie à $25^{\circ} \mathrm{C}$. En début d'expérience, la dureté totale de l'eau et le pourcentage en oxygène dissous sont respectivement $520 \mathrm{mg} . \mathrm{l}^{-1}$ et $37,5 \%$. Après $30 \mathrm{mn}$ et 60 mn d'exposition respectivement pour les stades L2 et L4, on détermine le nombre d'individus morts dans chaque pilulier pour calculer la $\mathrm{CL}_{50}$. Immédiatement après avoir dénombré les larves de Culicidés immobilisées, nous avons mesuré la dureté totale de l'eau (500 mg. $\left.1^{-1}\right)$ et le pourcentage en oxygène dissous $(75,7 \%)$ dans les piluliers correspondant à la plus faible concentration examinée pour laquelle toutes les larves ont été immobilisées pendant $60 \mathrm{mn}$. Cette concentration est de $0,05 \mathrm{mg} . \mathrm{l}^{-1}$ pour le stade L4 de C. pipiens.

\subsubsection{Lutte biologique: prédation par Gambusia affi-} nis

Le test de consommation de larves d'A.hispaniola au stade L4 par G.affinis a été réalisé à la température du laboratoire $\left(25^{\circ}-28^{\circ} \mathrm{C}\right)$ pendant le mois de mai 1996. Reddy (1975) et Otto $(1973,1974)$ ont utilisé les mêmes températures pour le test de l'intensité de prédation par G.affinis. Les larves sont testées en utilisant des milieux différents:

- eau brute du puits utilisée comme milieu témoin ;

- eau brute du gîte des poissons prélevée dans un bassin de la khettara Aourrad située dans la zone d'épandage des eaux usées de Marrakech (Milieu $\mathbf{M}_{1}$ );

- eau brute de la station Agadir Tachraft, gîte larvaire d'A. hispaniola. (Milieu $\mathrm{M}_{2}$ );

- eau diluée à 5 et $10 \%$ du deuxième bassin de lagunage, milieu où nous avons constaté la présence des larves de $C$. pipiens (Milieu $\mathrm{M}_{3}$,Tableau 1).

Les expériences ont été réalisées à raison de 20 larves par pilulier contenant chacun un poisson mâle ou femelle respectivement de 2,6 et 3 centimètres de taille. Le test est effectué séparément avec trois répétitions pour chaque milieu.

\section{Résultats}

\subsection{Toxicité létale du téméphos}

L'étude de l'effet létal du téméphos sur les larves d'A.hispaniola et de $C$. pipiens a montré que la $\mathrm{CL}_{50}$ (30mn) est respectivement égale à $21 \mu \mathrm{g} . \mathrm{l}^{-1}$ et $10 \mu \mathrm{g} . \mathrm{l}^{-1}$ pour le stade $\mathrm{L} 2$ (Fig.1 a-c). $\mathrm{La} \mathrm{CL}_{50}(60 \mathrm{mn})$ au stade L4 est de $23 \mu \mathrm{g} .1^{-1}$ pour A.hispaniola et de $12 \mu \mathrm{g} .1^{-1}$ pour C. pipiens (Fig.1 b-d). Au bout de $30 \mathrm{mn}$ d'exposition des larves au stade L4 au téméphos, on obtient un taux de mortalité inférieur à $50 \%$, ce qui n'a pas permis de calculer la $\mathrm{CL}_{50}(30 \mathrm{mn})$.

\subsection{Emploi de Gambusia affinis}

L'objectif de ce test est d'évaluer l'efficacité des Gambusies, pour éliminer les larves d'Anophèles.

Le taux de consommation par individu et par heure est d'une larve chez les mâles et de trois larves chez les femelles pour le milieu témoin (eau brute du puits). Il est d'une larve chez les mâles et de deux larves chez les femelles pour le milieu $M_{1}$. Pour le milieu $M_{2}$, il est de deux larves chez les mâles et de trois larves chez les femelles (Fig.2). Cependant, dans le milieu $\mathrm{M}_{3}$ dilué à $5 \%$, nous avons constaté que le taux de consommation de G.affinis est de quatre larves par heure chez les mâles et de 4 à 5 larves chez les femelles ; avec une dilution de $10 \%$ du milieu $\mathrm{M}_{3}$, ce taux est de deux larves chez les mâles et de trois larves chez les femelles (Fig. $3)$.

\section{Discussion}

Concernant la toxicité létale du téméphos utilisé comme larvicide sur les larves de $C$. fatigans et d'A. albimanus, Georghiou (1972) et Hurlbert (1975), ont trouvé des $\mathrm{CL}_{50}(24 \mathrm{~h})$ respectivement de $1,6 \mu \mathrm{g} . \mathrm{l}^{-1}$ et de $5 \mu \mathrm{g} .1^{-1}$.

Toutefois, les conditions d'utilisation des insecticides doivent être contrôlées pour éviter d'autres effets secondaires.

Hurlbert et al. (1972a) ont constaté que le traitement des étangs par l'insecticide chlorpyrifos (Dursban) réduisait de façon marquée l'abondance du zooplancton (Crustacés) et produisait ainsi une forte augmentation du phytoplancton. Cette prolifération algale, induite par l'insecticide, rend non seulement les étangs attractifs pour l'oviposition des moustiques mais aussi constitue un milieu nutritif enrichi pour les larves. 

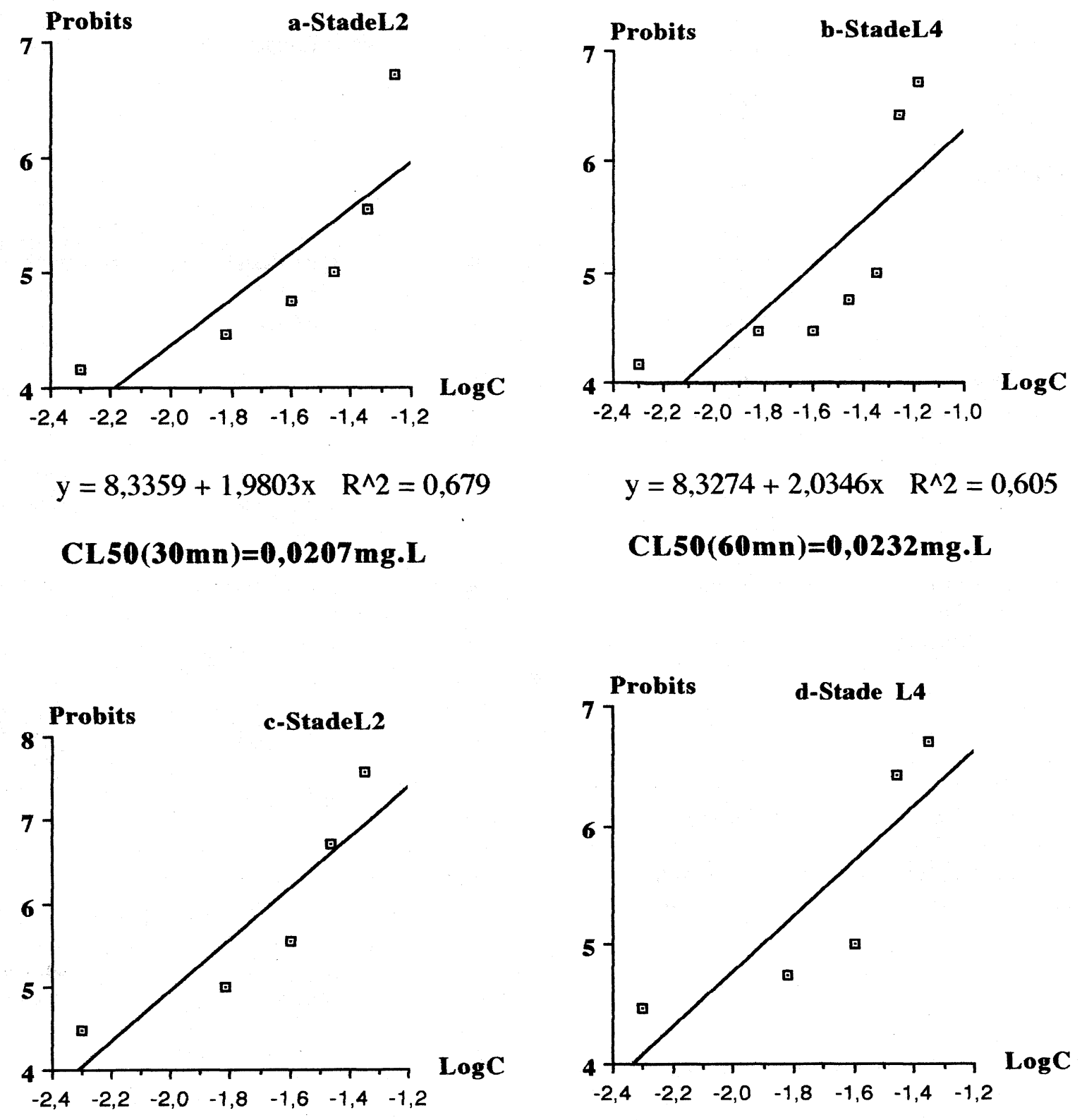

$y=11,054+3,0447 x \quad R^{\wedge} 2=0,808$ $y=9,4202+2,3167 x \quad R^{\wedge} 2=0,721$ CL50 $(30 \mathrm{mn})=0,0103 \mathrm{mg} \cdot \mathrm{L}$

CL50(60mn $=0,0124 \mathrm{mg} \cdot \mathrm{L}$

Fig.1. Toxicité létale du téméphos sur les stades larvaires L2 et L4 d'Anopheles hispaniola (a et b) et Culex pipiens (c et d). C: Concentration du téméphos.

Fig.1. Lethal toxicity of temephos on larval instars L2 and L4 of Anopheles hispaniola (a et b) and Culex pipiens (c et d.). C : Temephos concentration. 


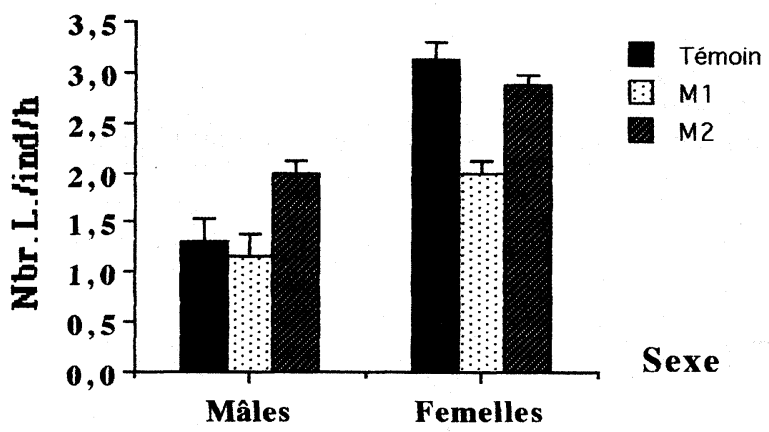

Fig. 2. Test de consommation de larves d'Anopheles hispaniola (stade L4) par un individu de Gambusia affinis et par heure, réalisé dans les milieux $\mathbf{M}_{1}$ et $\mathbf{M}_{2}$.

Fig. 2. Test of hourly consumption of Anopheles hispaniola larvae (instar L4) by an individual mosquitofish (Gambusia affinis) carried out in the media $M_{1}$ and $M_{2}$.

Nous avons pu noter une augmentation du taux d'oxygène de $38,2 \%$ en fin d'expérience sur la toxicité létale du téméphos. Le téméphos détruit totalement les larves de Culicidés brouteurs d'algues, ce qui augmente la production d'oxygène par photosynthèse.

Hoy et al. (1972) ont démontré que cet agent de contrôle, peut stimuler la production des moustiques. L'application aérienne de $0,014 \mathrm{~kg}$ de chlorpyrifos par hectare après 2 semaines réduit de $40 \%$ l'abondance des larves de C.tarsalis et $A$.freeborni. Mais après 6 à 10 semaines, les larves deviennent 2 à 3 fois plus abondantes dans les étangs traités.

El Kassimi (1990) avait constaté qu'un seul épandage de larvicide (téméphos) ne suffisait pas ; il doit être répété 7 fois dans un gîte donné à intervalle de 12 à 15 jours, soit 2 épandages par mois et 12 au maximum pour les 6 mois de l'année correspondant aux périodes de multiplication anophélienne.

Au laboratoire, la rémanence du téméphos est de 20 à 25 jours (Bouallam 1992). Celle, plus faible, observée par El Kassimi (1990) peut s'expliquer par le fait que sur le terrain, la qualité de l'eau du gîte est perturbée par plusieurs facteurs (courant d'eau, précipitations, crues).

Les stades larvaires L2 et L4 d'A.hispaniola sont plus résistants au téméphos que ceux de $C$. pipiens. De même, selon Georghiou (1972) et Hurlbert (1975), les larves d'A. albimanus sont plus résistantes au téméphos que celles de $C$. fatigans. Chez ces deux espèces, le stade L2 est plus sensible au téméphos que le stade

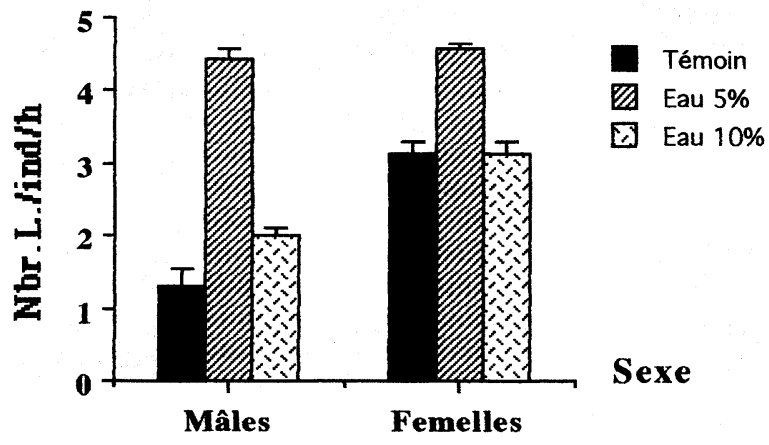

Fig. 3. Test de consommation de larves d'Anopheles hispaniola (stade L4) par un individu de Gambusia affinis et par heure, réalisé dans le milieu $\mathrm{M}_{3}$.

Fig. 3. Test of hourly consumption of Anopheles hispaniola larvae (instar L4) by an individual mosquitofish (Gambusia affinis) carried out in the medium $\mathrm{M}_{3}$.

$\mathrm{L} 4$, ce qui peut traduire une résistance acquise au cours de la croissance larvaire. Il est donc intéressant de chercher l'explication biologique de cette résistance pour proposer d'autres moyens de lutte plus efficaces et moins dangereux pour l'environnement.

Ayant une action très efficace sur les premiers stades larvaires, le téméphos, à faible concentration, agit sur les stades L1 et L2 qui sont moins résistants. Ceci permet d'utiliser des quantités plus faibles que celles nécessaires pour lutter contre les stades L4.

Le téméphos n'a pas d'action sur les nymphes quelles que soient les concentrations utilisées. A ce stade, l'organisme ne se nourrit pas alors que le téméphos agit par ingestion.

L'utilisation de G.affinis est souvent efficace. Hurlbert et al. (1972b) ont constaté que les larves de Chironomidés (Diptera) atteignent une densité exceptionnelle dans le sédiment de petites pièces d'eau sans G.affinis . Par contre, ces diptères sont éliminés en décembre dans des pièces d'eau avec G.affinis .

La faune culicidienne est très réduite dans les lieux occupés par les fortes densités en G.affinis qui se nourrissent des larves et des alevins de leurs commensaux réduisant peu à peu ces populations jusqu'à leur disparition (Teton 1976).

Bay \& Anderson (1966, in Hurlbert et al.1972b) ont constaté que la prédation d'organismes benthiques par G.affinis serait moins intensive dans les étendues d'eaux plus importantes (étangs et mares). 
Hoy et al. (1972) et Hulbert et al. (1972b) ont démontré que G.affinis peut stimuler la production des moustiques dans les rizières en affectant la population des invertébrés qui s'en nourrissent tels les Notonectidés (Farley \& Younce 1977, Hall et al. 1970). En effet, selon Hoy et al. (1972), l'introduction de G.affinis dans les étangs à raison de $0,22 \mathrm{~kg} \cdot \mathrm{ha}^{-1}$ provoque, après 2 semaines, une augmentation de l'abondance des larves de moustiques. Après 10 semaines, l'abondance décroît progressivement jusqu'à atteindre celle des étangs témoins. Après introduction de G.affinis à une biomasse élevée $\left(0,67 \mathrm{~kg}_{\text {gha }}{ }^{-1}\right)$, d'autres étangs développeraient peu de moustiques durant toute l'expérience par rapport aux étangs témoins.

Le taux de consommation des larves d'A.hispaniola au stade $\mathrm{L} 4$ est très élevé dans le milieu $\mathrm{M}_{3}$ surtout quand l'eau est diluée à $5 \%$.

Le taux de consommation par G.affinis varie en fonction des milieux mais reste toujours plus élevé chez les femelles que chez les mâles. Ceci peut être expliqué par le fait qu'avant la reproduction les femelles ont un appétit plus important, alors que durant la gestation, elles se nourrissent moins. Le taux peut varier aussi en fonction de la taille des mâles $(2,6 \mathrm{~cm})$ et des femelles $(3 \mathrm{~cm})$.

\section{Conclusion}

La lutte antilarvaire chimique par le téméphos a montré que l'action est efficace sur les premiers stades larvaires. Le stade L 2 est plus sensible que le stade L4, ce qui peut être attribué à une résistance acquise au cours de la croissance larvaire. Chez A.hispaniola (vecteur potentiel du paludisme), les stades L2 et L4, sont plus résistants que chez $C$. pipiens. Cette résistance a probablement été acquise, suite à l'épandage de ce larvicide qui a duré jusqu'en septembre 1988 dans la région de Marrakech.

L'emploi de poissons larvivores présente des avantages certains dans la lutte biologique. Le taux de consommation par G.affinis varie en fonction des milieux mais reste toujours plus élevé chez les femelles que chez les mâles.

Cependant, les conditions de cette utilisation doivent être mieux définies pour éviter les effets néfastes sur la chaîne alimentaire.

\section{Travaux cités}

Al-Daham N.K., Hug M.F. \& Sharma K.P. 1977. - Notes on the ecology of fishes of the genus Aphanius and Gambusia affinis in southern Iraq. Freshwater. Biol., 7 : 245-251.
Bouallam S. 1992. — Le Paludisme et les Moustiques dans la région de Marrakech. Ecologie et Cycles biologiques des espèces culididiennes.Thèse 3 ème cycle, Univ. Cadi Ayyad, Fac Sc. Semlalia, Marrakech : $127 \mathrm{p}$.

El Kassimi M.B. 1990. - Le paludisme dans la province de Chefchaouen de 1983 à 1987. Thèse Médecine Rabat, $N^{\circ} 35$.

Farley D.G. \& Younce L.C. 1977. — Effects of Gambusia affinis (Baird \& Girard) on selected nontarget organisms in Fresno County rice fields. Proc. Pap. Calif. Mosquito Control Assoc., 45 : 87-94.

Georghiou G.P. 1972. - Studies on resistance to carbamate and organophosphorus insecticide in Anopheles albimanus Amer. J. Trop. Med. Hyg., 21 : 797.

Guy Y. 1963. - Bilan épidémiologique du paludisme au Maroc (données receuillies en 1960, 1961 et 1962). Ann. Parasit. Hum. Comp., 38 : 823-857.

Hall D.J., Cooper W.E. \& Werner E.E. 1970. - An experimental approach to the production dynamics and structure of freshwater animal communities. Limnol. Oceanogr., 15 : 839-928.

Hoy J.B., Kaufmann E.E. \& O'Berg A.G. 1972. - A large-scale field test of Gambusia affinis and chloropyritus for mosquito control. Mosqui. News, 32 : 161-171.

Hurlbert S.H 1975. - Secondary effects of pesticides on aquatic ecosystems. Residue Reviews, 58 : 81-148.

Hurlbert S.H. \& Mulla M.S.1981. - Impacts of mosquitofish (Gambusia affinis) predation on plankton communities. Hydrobiologia, $83: 125-151$.

Hurlbert S.H., Mulla M.S. \& Willson H.R. 1972a. — The effects of anorganophosphorus insecticide on the phytoplankton, zooplankton, and insect populations of freshwater pond. Ecol. Monogr., 42 : 269- 299.

Hurlbert S.H., Zedler J. \& Fairbanks D. 1972b. - Ecosystem alternation by mosquitofish (Gambusia) predation. Science, 175 : 639641.

Kline D.L., Hagan D .V. \& Wood J.R.1994. — Culicoides responses to 1- octen-3-ol and carbon dioxide in salt marshes near sea Island, Georgia, USA. Med. Vet. Ent., 8 : 25-27.

Krumholtz L.A. 1948. - Reproduction in the western mosquitofish Gambusia affinis. (Baird \& Girard) and its use in mosquito control. Ecol. Monogr., $18: 1-43$.

Labounty J.T. \& Deacon J.E. 1972. - Cyprinodon milleri, a new species of pupfish (Family Cyprinodontidae) from Death Valley, California. Copeia , 1972: 769-780.

Le Sueur D., Sharp B.L., Fraser C. \& Ngxongo S.M. 1993. - Assessment of the residual efficacy of Lambda- Cyhalothrin 1. A laboratory study using Anopheles arabiensis and Cimex lectularius (Hemiptera : Cimicidae) on treated daub wall substrates from natal, South Africa. J. Amer. Mosqu. Control Association, 9 (4) : 408-413.

Losos B. \& Hetesa J. 1973. - The effect of mineral fertilization and of carp fry on the composition and dynamics of plankton. Hydrobiol. Stud. (Prague) 3 : 173-217.

Ministère de la santé publique, Maroc 1981. — Lutte antipaludique, guide des activités. Direction affaires techniques, Maroc .

Otto R.G. 1973. - Temperature tolerance of the mosquitofish. J.Fish.Biol., 5 : 575-585.

Otto R.G. 1974. - The effects of acclimation to cyclic thermal regimes on heat tolerance of Western mosquitofish. Trans. Amer. Fish. Soc., 103 : 331-335.

Reddy S.R. 1975. - Effect of water temperature on the predatory efficiency of Gambusia affinis. Experientia, 31 : 801-802. 
Sharp B.L., Le Sueur D., Wilken G.B., Bredenkamp B.L.F., Ngxongo S. \& Gouws E. 1993. - Assessment of the residual efficacy of Lambda- Cyhalothrin 2. A comparison with DDT for the intradomiciliary control of in South Africa. J. Amer. Mosqu. Control Association, $9(4)$ : 414-420.

Sicault G. 1934. - Note sur l'adaptation du Gambusia holbrooki aux eaux salées. Bull. Soc. Pathol. exot. 27 : 485-588.

Sinegre G., Jullien J.L \& Crespo O. 1976. - Résistance de certaines populations de Culex pipiens (L.) au chlorpyrifos (Dursban ${ }^{\mathrm{R}}$ ) en languedoc-Roussillon (France).Cah. O.R.S.T.O.M., Ser. Ent. Méd. et Parasitol., XIV (1) : 49-59.

Teton J. 1976. - Gambusia affinis, un bienfaiteur américain presque oublié. Arch. Hydrobiol., 35 :11-14.

Tietze N.S., Olson M.A., Hester P.G. \& Moore J.J.1993. - Tolerance of sewage treatment plant micro-organisms to mosquitocides. J. Amer. Mosqu. Control Association, 9 (4) : 477-479.
Vanessen P.H.A., Kemme J.A, Ritchie S.A. \& Kay B.H.1994. Differential responses of Aedes and Culex mosquitoes to octenol or light in combination with carbon dioxide in Queensland, Australia. Med. Vet. Ent., 8 (1) : 63-66.

Vulvule J.M., Beach R.F., Atieli F.K., Roberts J.M., Mount D.L. \& Mwangi R.W.1994. - Reduced susceptibility of Anopheles gambiae to permethrin associated with the use of permethrin impregnated bednets and curtains in Kenya. Med. Vet. Ent., 8 (1) : 71-74.

Zaim M., Zahirnia A.H. \& Manouchehri A.V. 1993. - Survival rates of Anopheles culicifacies S.L. and Anopheles pulcherrimus in sprayed and unsprayed villages in Ghassreghand district, Balluchistan, Iran, 19911. J. Amer. Mosqu. Control Association, 9 (4) : 421-425. 